\title{
Intermédialités
}

Histoire et théorie des arts, des lettres et des techniques

Intermediality

History and Theory of the Arts, Literature and Technologies

\section{Articulation et rythme : matière, pensée et création dans le discours}

\section{Lucie Bourassa}

Numéro 16, automne 2010

rythmer

rhythmize

URI : https://id.erudit.org/iderudit/1001962ar

DOI : https://doi.org/10.7202/1001962ar

Aller au sommaire du numéro

\section{Éditeur(s)}

Revue intermédialités (Presses de l’Université de Montréal)

ISSN

1705-8546 (imprimé)

1920-3136 (numérique)

Découvrir la revue

Citer cet article

Bourassa, L. (2010). Articulation et rythme : matière, pensée et création dans le discours. Intermédialités / Intermediality, (16), 185-206.

https://doi.org/10.7202/1001962ar
Résumé de l'article

Cet article propose un rapprochement et une confrontation entre la notion de rythme d'Henri Meschonnic et celle d'articulation (Artikulation; Gliederung) de Wilhelm von Humboldt. Ces notions présentent plusieurs parentés, bien qu'elles s'inscrivent dans des contextes épistémologiques différents et qu'elles ne soient nullement réductibles l'une à l'autre. On les compare ici en particulier sur le plan de l'enracinement du langage dans le corps et du rôle central joué par le signifiant dans la pensée. Ce rapprochement se veut un parcours heuristique pour repenser le rythme dans le discours. On essaie d'abord de donner une sorte de profondeur à la notion de Meschonnic, c'est-à-dire d'en éclairer certains aspects sous un autre jour : avec la notion d'articulation, on devrait mieux comprendre, en particulier, comment le rythme permet de dépasser la représentation instrumentale du langage. On reformule ensuite la définition du rythme à la lumière du concept d'articulation, lequel se révèle utile en particulier pour en considérer la dimension sensible, perceptible, que la théorie de Meschonnic laisse de côté.
Ce document est protégé par la loi sur le droit d'auteur. L'utilisation des services d'Érudit (y compris la reproduction) est assujettie à sa politique d'utilisation que vous pouvez consulter en ligne.

https://apropos.erudit.org/fr/usagers/politique-dutilisation/ 


\title{
Articulation et rythme: matière, pensée et création dans le discours
}

\author{
Lucie Bourassa
}

\author{
La liaison indissoluble de la pensée, \\ des organes de la voix et de l'ouie avec le langage \\ se trouve irrévocablement dans l'organisation \\ originaire de la nature humaine, \\ qui ne peut être expliquée davantage ${ }^{1}$.
}

(Wilhelm von Humboldt)

\begin{abstract}
e curieux phénomène qu'on appelle rythme, en apparence simple et évident, mais complexe et fuyant dès qu'on essaie de le définir, m’occupe depuis longtemps. Cet intérêt m'est venu de la lecture de la poésie moderne et contemporaine, ainsi que de longues années de pratique et d'écoute musicales. La poésie me retenait moins par ses images ou ses thèmes que par ce qu'elle faisait avec le langage, en particulier par la manière dont le discours s'y déroulait, dans sa dimension physique, signifiante et dynamique. Ce déploiement, c'était le rythme en fait, mais pas tel qu'on le comprenait habituellement dans les études
\end{abstract}

1. «Die unzertrennliche Verbindung des Gedanken, der Stimmwerkzeuge und des Gehörs zur Sprache liegt unabänderlich in der ursprünglichen, nicht weiter zu erklärenden Einrichtung der menschlichen Natur.» Wilhelm von Humboldt reprend cette phrase deux fois, dans Ueber die Verschiedenheiten des menschlichen Sprachbaues (Wilhelm von Humboldt, Gesammelte Schriften, 17 volumes, Édition Albert Leitzmann et al., Berlin, Behr, 1903-1936, vol. VI, p. 154) et Ueber die Verschiedenheit des menschlichen Sprachbaues und ihren Einfluss auf die geistige Entwicklung des Menschengeschlechts (Humboldt, 1903-1936, vol. VII, p. 53). À moins d'une indication contraire, c'est moi qui ai traduit les passages de Humboldt que je cite. Et quand je ne citerai pas une autre traduction, je renverrai aux œuvres de Humboldt d'après l'édition Humboldt, 1903-1936, en mentionnant le numéro du volume et de la page. 
littéraires, où on l'identifiait à une métrique. J'ai alors tenté de voir comment on pouvait définir le rythme et étudier ses manifestations dans le discours sans le renvoyer à la mesure. Henri Meschonnic avait, une dizaine d'années plus tôt, proposé un travail considérable qui allait dans ce sens², auquel je dois beaucoup. Cette théorie laisse cependant de côté la dimension perceptive du rythme, qui m'apparaissait au contraire importante: j'ai donc repensé la notion, en faisant appel à l'antique concept d'esthésis - qui lie le sensible et l'intelligible ${ }^{3}$-, et en le définissant à partir de la temporalité et d'une dynamique de tensions. ${ }^{4}$. Dans les pages qui suivent, je voudrais apporter un autre éclairage à la question du rythme dans le discours, en mettant cette fois en relation la théorie de Meschonnic avec la notion d'articulation telle que l'avait pensée Wilhelm von Humboldt.

\section{RYTHME ET ARTICULATION, CONTRE LE SIGNE}

Dans le domaine de la poétique, l'apport de Meschonnic aux réflexions sur le rythme est l'un des plus décisifs des dernières décennies. Le rythme est le concept central de sa théorie, et il acquiert un statut épistémologique exceptionnel. Au-delà de ses applications possibles aux textes littéraires, la poétique de Meschonnic se veut aussi, selon le titre et le sous-titre de son grand œuvre, une «Critique du rythme» et une «Anthropologie historique du langage». Son enjeu ne consiste pas simplement à examiner les théories du rythme, mais également à critiquer, en prenant le rythme comme point de départ, les théories du langage qui sous-tendent les sciences humaines et la philosophie. Critique du rythme présente par ailleurs un caractère décapant et novateur: ce livre remet en cause la plupart des idées reçues sur le rythme et propose une nouvelle notion qui l'assimile à une organisation du mouvant, sans référence à la mesure. Ainsi défini, le rythme concerne tout le discours.

2. Henri Meschonnic, Critique du rythme. Anthropologie historique du langage, Lagrasse, Verdier, 1982.

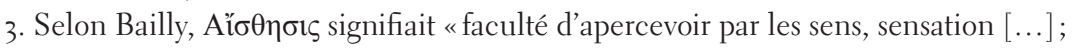
faculté de percevoir par l'intelligence, action de s'apercevoir ». Anatole Bailly, Dictionnaire grec-français, édition revue, Paris, Hachette, 1950, p. 49.

4. On trouvera dans Lucie Bourassa, Rythme et sens. Des processus rythmiques en poésie contemporaine, Montréal, Éditions Balzac, 1993, cette réflexion théorique sur la notion de rythme, qui se développe en plusieurs étapes. Dans un ouvrage plus récent, j’ai beaucoup approfondi la question des liens entre rythme et temporalité: Lucie Bourassa, L'entrelacs des temporalités. Du temps rythmique au temps narratif, Québec, Nota bene, 2009. 
Au centre de cette critique se trouve la vision platonicienne du rythme comme forme «déterminée par une "mesure" et assujettie à un ordre" ». Outre son caractère réducteur, cette notion métrique fait du rythme une forme séparée du sens, pour le limiter à une fonction ornementale ou expressive. Opposé à toute vision dualiste du langage, Meschonnic repense le rythme comme organisation de marques et du sens du sujet dans son discours:

Le rythme, organisation des marques dans le discours, est l'organisation du sens, mais aussi de la force, dans le discours. Une force inséparable de sa syntagmatique et de sa paradigmatique. En quoi il fait la contestation de ce que Saussure appelait les «subdivisions traditionnelles», lexique, morphologie, syntaxe - catégories de la langue, non du discours. Le rythme n'est plus une forme à côté du sens. Et comme le sens est une activité des sujets - activité sociale, historique, spécifique -, le rythme est une organisation du sujet dans et par son discours. Le rythme inclut alors non seulement les alternances mesurables d'accents, mais toute la prosodie, effets d'échos consonantiques et vocaliques, ainsi que l'intonation, pour le parlé. Il reprend, dans leur relation empirique, le corporel et le social que la linguistique de l'énoncé et de la phrase, même la linguistique du discours, jusqu'ici abandonnaient à l'extralinguistique 6 .

Pour forger cette définition - dont figurait une première version, quelque peu différente, dans Critique du rythme -, il s'est inspiré d'une acception présocratique de la notion de rhuthmos, telle que l'a mise au jour Émile Benveniste, " "configuration particulière du mouvant", ou "arrangement caractéristique des parties dans un tout", "forme du mouvement" ". Fort stimulante du fait qu'elle tient ensemble les aspects corporels, énonciatifs et sociaux du discours, la définition de Meschonnic n’est pas explicite sur la manière dont les «marques » qu'il énumère donnent au mouvement une «configuration particulière ».

Meschonnic refuse de considérer cette dimension perceptive, parce qu'il s'agit selon lui d'une question de psychologie qui ne tient pas compte de la spécificité du langage, et surtout qui reconduit le «dualisme» ou la «métaphysique du signe », la séparation du son et du sens. Or, sa critique du rythme s'inscrit précisément contre cette représentation sémiotique du langage, qui assimile ce

5. Voir Émile Benveniste, «La notion de rythme dans son expression linguistique », Problèmes de linguistique générale, tome 1, Paris, Gallimard, coll. "Bibliothèque des sciences humaines ", 1966, p. 334. Benveniste paraphrase la définition de Platon dans Lois (665a).

6. Gérard Dessons et Henri Meschonnic, Traité du rythme. Des vers et des proses, Paris, Dunod, 1998, p. 75.

7. Meschonnic, 1982, p. 70. 
dernier à une collection de signes discontinus - simples substituts des choses ou d'idées existant en dehors de lui. Refuser une telle vision, c'est aussi mettre à mal une «anthropologie conçue sur le modèle binaire du signe ${ }^{8}$ ». Le "paradigme du signe» est problématique en ce qu'il entraîne une vision discontinue des pratiques humaines, et «des objets qu'il décrit ${ }^{9}$ », alors que :

$[\mathrm{L}] \mathrm{a}$ théorie nouvelle du rythme doit reconnaître, $[\ldots]$ tout ce qu'il y a $[\ldots]$ dans le langage et qui est de l'ordre du continu, entre le corps et le langage, entre une langue et une littérature, entre une langue et une pensée: un ensemble de dispositifs signifiants qui sont des sémantiques du continu ${ }^{10}$.

Dans l'élaboration de sa poétique, Meschonnic fait très souvent appel à Humboldt, à qui il donne un rôle fondateur: «Humboldt est sans doute le premier et peut-être le seul à avoir fait une théorie du langage qui soit une anthropologie ${ }^{11}$.» Il lui a consacré plusieurs études ${ }^{12}$, mais il ne s'intéresse guère, sauf erreur de ma part, au concept de l'articulation. Je n'y ai trouvé qu'une seule mention de cette notion dans ses textes sur Humboldt: «Le son articulé est ce qui fait le sens ${ }^{13}$.» Pourtant, l'articulation joue dans l'œuvre de Humboldt un rôle analogue à celui du rythme dans la sienne: chacun de ces concepts figure au centre d'une théorie du langage, et il en constitue le «terme anti-sémiotique », comme le montre Jürgen Trabant ${ }^{14}$. Il faut cependant mentionner que cette théorie de l'articulation se développe peu à peu dans les écrits de Humboldt,

8. Dessons et Meschonnic, 1998, p. 35. Le signe est en effet aux yeux de Meschonnic l'emblème d'une série de dualismes qui fonctionnent de manière solidaire, dans les domaines linguistique, anthropologique, philosophique, théologique, social, politique, etc. Voir Henri Meschonnic, Politique du rythme, politique du sujet, Lagrasse, Verdier, 1995, p. 114-116. Voir aussi "Critique du signe», dans Lucie Bourassa, Henri Meschonnic. Pour une poétique du rythme, Paris, Bertrand-Lacoste, 1997, p. 40-45.

9. Dessons et Meschonnic, 1998, p. 37.

10. Ibid., p. $3^{8 .}$

11. Meschonnic, 1982, p. 47.

12. La première d'entre elles, «Humboldt et le sens du langage » (Henri Meschonnic, Le signe et le poème, Paris, Gallimard, coll. «Le chemin», 1975, p. 123-139), fait suite à la lecture de la première traduction importante de l'œuvre de Humboldt en France (Wilhelm von Humboldt, Introduction à l'œuvre sur le kavi et autres essais, trad. Pierre Caussat, Paris, Éditions du Seuil, coll. «L’ordre philosophique», 1974).

13. Meschonnic, 1975, p. 134.

14. Jürgen Trabant, «Le Humboldt d'Henri Meschonnic», dans Gérard Dessons, Serge Martin et Pascal Michon (dir.), Henri Meschonnic, La pensée et le poème, Paris, In Press Éditions, 2005, p. 179. 
et qu'il n'y a pas de texte qui lui soit entièrement et explicitement consacré. La version la plus développée de cette théorie se trouve dans un essai grammatologique sur l'écriture alphabétique ${ }^{15}$. On doit à Trabant d'avoir retracé l'histoire de cette notion et d'avoir montré son importance dans la théorie du langage de Humboldt ${ }^{16}$.

L'articulation (Artikulation, Gliederung ${ }^{17}$ ) est un principe de division et de réunion commun au son et au concept, qui ne se produisent pas l'un sans l'autre et agissent réciproquement l'un sur l'autre :

À l'acte de l'entendement qui produit l'unité du concept correspond, comme son signe sensible, l'unité du mot; et tous deux dans la pensée doivent être associés par le discours aussi intimement que possible. Car comme la puissance de la réflexion produit la séparation et l'individualisation des sons au moyen de l'articulation, celleci doit, à son tour, agir sur le contenu de la pensée de façon séparatrice et individualisante; l'articulation doit lui permettre, partant de l'indissocié et aspirant à l'indissocié, à l'unité absolue, de refaire ce trajet à travers la séparation ${ }^{18}$.

Plus encore, elle s'étend à tout le langage, dont elle est «l'essence», puisque tout en lui est séparation et liaison:

[L]'articulation est justement l'essence du langage; il n'y a rien en lui qui ne puisse être partie et tout, l'efficacité de son activité continue repose sur la légèreté, l'exactitude et la concordance de ses séparations et ses compositions. Le concept de l'articulation est sa fonction logique, ainsi que celle de la pensée même ${ }^{19}$.

15. Ueber die Buchstabenschrift und ihren Zusammenhang mit dem Sprachbau [1824], dans Humboldt, 1903-1936, vol. V, p. 107-133.

16. Voir en particulier Jürgen Trabant, Artikulationen. Historische Anthropologie der Sprache, Frankfurt am Main, Suhrkamp, 1998, en particulier le chapitre intitulé «Artikulieren», p. 67-87, ainsi que «Von Schrei zur Artikulation: Über der Ursprung der Sprache », dans Jürgen Trabant, Was ist Sprache?, Münich, C. H. Beck Verlag, 2008, p. 25-51. J'ai traduit les passages des textes allemands de Trabant que je cite.

17. Ces deux mots (Artikulation, Gliederung) désignent le phénomène de l'articulation chez Humboldt, qui les emploie dans des sens un peu différents, le premier renvoyant généralement à la phonation, le second au découpage intellectuel, ou au phénomène dans son ensemble. Je renvoie aux essais de Trabant cités dans la note précédente.

18. Wilhelm von Humboldt, "Sur l'étude comparée des langues dans son rapport aux différentes époques du développement du langage», dans Sur le caractère national des langues et autres écrits sur le langage, trad. Denis Thouard, Paris, Éditions du Seuil, coll. «Points. Essais », 2000, p. 93.

19. «Die Gliederung ist aber gerade das Wesen der Sprache; es ist nichts in ihr, das nicht Theil und Ganzes seyn könnte, die Wirkung ihres beständigen Geschäfts beruht auf der Leichtigkeit, Genauigkeit und Uebereinstimmung ihrer Trennungen und 
Or, les notions d'articulation et de rythme, telles qu'elles sont définies par Humboldt et Meschonnic, présentent plusieurs parentés, bien qu'elles s'inscrivent dans des contextes épistémologiques différents et qu'elles ne soient nullement réductibles l'une à l'autre ${ }^{20}$. Je les comparerai ici en particulier sur le plan de l'enracinement du langage dans le corps et du rôle central joué par le signifiant. Ce rapprochement se veut un parcours heuristique pour repenser le rythme. Je compte d'abord donner une sorte de profondeur à la notion de Meschonnic, éclairer certaines questions sous un autre jour: avec la notion d'articulation, on devrait mieux comprendre, en particulier, comment le rythme permet de dépasser la représentation instrumentale du langage. Je me propose ensuite de retravailler la définition du rythme à la lumière de l'articulation, qui se révèle utile pour considérer la dimension esthésique d'une configuration particulière du mouvant.

\section{DU CORPS À L'ANTHROPOLOGIE HISTORIQUE}

Humboldt a été l'un des premiers à reconnaître pleinement la fonction cognitive du langage et des langues: "L'activité intellectuelle et la langue ne font [...] qu'un, sont inséparables l'une de l'autre; on ne peut même pas considérer tout simplement la première comme productrice et la seconde comme produit ${ }^{21}$.» Dans sa théorie, cette unité de la pensée et du langage dépend du corps, parce qu'elle ne peut s'accomplir que grâce à la profération de sons : «Le son articulé,

Zusammensetzungen. Der Begriff der Gliederung ist ihre logische Function, so wie die des Denkens selbst.» Humboldt, 1903-1936, vol. V, p. 122.

2o. Jürgen Trabant rapproche implicitement ces notions alors qu'il reprend le soustitre de Critique du rythme pour son livre Artikulationen. Historische Anthropologie der Sprache, sans développer la comparaison. Dans ce livre, Trabant reprend et enrichit la notion humboldtienne pour la mettre au centre de sa propre réflexion sur le langage et il veut, à l'exemple de Meschonnic, faire de la réflexion sur le langage « une anthropologie historique, c'est-à-dire inscrire la théorie du langage dans une vision de l'homme qui considère comme fondamentale son historicité et rattache celle-ci au langage - contre toutes les tentations naturalistes et universalistes» («so möchte ich doch mit Meschonnic die Theorie des Sprache gleichsetzen mit einer historischen Anthropologie der Sprache, d. h. die Theorie der Sprache einschreiben in ein Denken des Menschen, das dessen Historizität für grundlegend hält und das diese gerade an der Sprache festmacht - gegen alle naturalistischen und universalistischen Anfechtungen.»). Trabant, 1998, p. 11.

21. «Die intellectuelle Thätigkeit und die Sprache sind [...] Eins und unzertrennlich von einander; man kann nicht einmal schlechthin die erstere als das Erzeugende, die andre als das Erzeugte ansehen.» Humboldt, 1903-1936, vol. VI, p. 152. 
ou, pour parler de manière plus générale, l'articulation, est l'essence propre de la langue, le levier grâce auquel elle et la pensée se réalisent, la clé de voûte de leur intime union réciproque ${ }^{22}$. » En fait, ce qui compte n'est pas d'abord le son, mais l'articulation comme mouvement physique. Pour illustrer cela, Humboldt donne l'exemple des sourds-muets qui peuvent apprendre à parler quand on leur montre comment placer et bouger leur appareil phonatoire. Il observe qu'il s'opère en eux « une remarquable segmentation des sons articulés ${ }^{23}$ ». Car l'esprit a le pouvoir de contraindre les organes de la parole «à un traitement des sons [...] qui corresponde à la forme de son action ${ }^{24} »$; le lien entre la forme d'action de l'esprit (la pensée, la réflexion) et la prononciation consiste dans le fait que les deux sont organisées par un principe de division et de liaison, par l'articulation dans le sens le plus général de la notion. Celle-ci est définie de la manière la plus détaillée dans l'essai l'écriture alphabétique, où Humboldt théorise, bien avant André Martinet, la «double articulation ${ }^{25}$ » : la première étant la division du monde et des idées en unités de contenu (sémantiques), la seconde, la division des séquences sonores qui constituent ces unités de contenu en unités distinctives (phonologiques). Dans Verschiedenheiten, l'exemple des sourds-muets a permis à Humboldt d'illustrer ce qu'il appelle la «face intellectuelle et en quelque sorte intérieure $^{26}$ » du son; on dirait maintenant sa dimension phonologique. Après avoir discuté cet exemple, il revient cependant sur le son, qui demeure malgré tout pour lui fondamental: Humboldt a une conception premièrement acoustique - vocale, auditive et dialogique - du langage.

La pensée par le langage est donc corporelle en son fondement, qui est l'articulation de la voix et sa réception par l'oreille. Mais en même temps, les sons de la parole sont irréductibles aux sons naturels. Dès le premier texte où il aborde la question des liens entre la pensée et le langage, «Über Denken und Sprechen ${ }^{27}$ », Humboldt oppose «la nature et la structure cognitive-articulatoire

22. «Der articulierte Laut oder, allgemeiner zu sprechen, die Articulation ist das eigentliche Wesen der Sprache, der Hebel, durch welchen sie und der Gedanke zu Stande kommt, der Schlussstein ihrer beiderseitigen innigen Verbindung. » Ibid.

23. «Eine merkwürdige Zerlegung des articulierten Lautes», ibid., p. 153.

24. «Die Articulation beruht auf der Gewalt des Geistes über die Sprachwerkzeuge, sie zu einer Behandlung des Tons zu nöthigen, welche der Form seines Wirkens entspricht. » Ibid., vol. VI, p. 152.

25. Voir à ce sujet Trabant, 1998, p. 79-85.

26. «Um aber den articulirten Laut [...] von seiner intellectuellen, gleich innerlichen Seite zu zeigen [...]», Humboldt, 1903-1936, vol. VI, p. 154-155.

27. Ce texte date de 1795 . 
du son de la langue avec le "son de la nature", le "cri des passions"28 ». Selon Trabant, ce trait distingue de manière décisive la théorie humboldtienne d'autres grands textes du $18^{\mathrm{e}}$ siècle - ceux de Condillac, de Rousseau et de Herder - qui ont essayé de décrire l'articulation dans une perspective diachronique, en imaginant les étapes qui ont précédé l'apparition du langage verbal, sans arriver à expliquer le saut vers la dimension conceptuelle de la parole. Humboldt ne cherche pas à comprendre de manière génétique, historique, l'origine du langage humain ${ }^{29}$. Le dispositif originaire qu'il décrit est un fonctionnement, et celui-ci est déjà cognitif, les sons ayant des propriétés intellectuelles. Seul à se composer d'unités élémentaires pouvant se combiner de multiples manières, le son du langage est proprement anthropologique:

[I]l n'existe pas autrement de tels sons [constitués d'éléments autorisant des compositions multiples] dans toute la nature, parce qu'aucun être, outre l'homme, n'invite ses semblables à la compréhension par le penser-ensemble, les autres ne peuvent au mieux qu'inviter à l'action par le sentir-ensemble ${ }^{30}$.

La théorie de Meschonnic ne veut pas expliquer le langage comme «organisation originaire de la nature humaine». Pour lui, l'enjeu de la notion de rythme est d'abord, avec la critique du signe et la recherche d'une sémantique du continu, un moyen de reconnaître la subjectivité et l'historicité des discours. À l'exemple de l'articulation, le rythme s'enracine dans le corps, il est même «le gardien du corps dans le langage ${ }^{31}$ ». La présence du corps dans le langage est l'un des objets de la critique du rythme: "C'est parce que le langage ne s'enlève pas du corps, et qu'il y a à chercher ce qui en reste dans le discours, que le langage relève non seulement des sciences du langage, de la théorie du langage, mais encore d'une anthropologie ${ }^{32}$.» Et Meschonnic fait l'hypothèse que «le corps est plus engagé dans le langage quand le rythme joue un rôle plus grand ${ }^{33}$ ». Sauf que, dans l'écrit en particulier, il est difficile «de savoir ce qui reste du corps» dans un texte, de savoir quel rôle joue le corps « dans l'organisation du langage en tant que telle ${ }^{34}$ ».

28. Voir à ce sujet Trabant, 2008, p. 39.

29. Voir ibid., p. 46.

30. «Solche Töne giebt es sonst in der ganzen übrigen Natur nicht, weil niemand, ausser dem Menschen, seine Mitgeschöpfe zum Verstehen durch mitdenken, sondern höchstens zum Handeln durch Mitempfinden einladet. » Humboldt, 1903-1936, vol. VII, p. 583 , cité par Trabant, 2008, p. $3^{8 .}$

31. Meschonnic, 1982, p. 651 .

32. Ibid.

33. Ibid., p. 655.

34. Ibid. 
Dans Critique du rythme ${ }^{35}$, il passe en revue plusieurs théories qui ont voulu éclairer ces relations entre le langage, le rythme et le corps, par exemple rattachant le rythme du discours à des périodicités physiologiques (cœur, respiration), à des mouvements du corps et aux pulsions. Tout comme Humboldt distinguait l'articulation langagière des sons produits par les animaux, et refusait de chercher l'origine du langage dans la nature, Meschonnic défend l'historicité «radicale» du rythme et du discours. Et il donne à Humboldt la paternité d'un tel point de vue: «Il est l'initiateur de ce qui fait que les discours sur l'origine du langage prennent, irréversiblement pour nous, l'air des mythes anciens ${ }^{36}$. » Affirmer que le rythme est le gardien du corps dans le discours n’équivaut donc pas, pour lui, à en faire le dérivé des rythmes physiologiques divers. Il critique ainsi de nombreuses théories qui ont privilégié cette voie: «Le physiologisme oublie que le corps est un corps social $[\ldots]^{37}$. »

Fait intéressant, plusieurs des théories qu'il commente établissent un lien entre le rythme poétique et l'articulation - mais seulement au sens physique de celle-ci. Maurice Grammont avait fait l'hypothèse d'une association entre la physique de la parole (les propriétés articulatoires et auditives des timbres, des accents et de l'intonation) et la signification (émotions, idées), par le biais de corrélations synesthésiques. Ses idées ont été reprises et développées par André Spire, qui croit que les mots ont le pouvoir de nous rappeler des sensations passées, liées notamment à l'apprentissage du langage. L'audition des mots pourrait ébranler tout notre système physiologique, en partant de l'appareil phonatoire et en s'étendant au «cou », à la «tête », au «visage» et au corps entier. Le poète retrouverait une telle «danse laryngo-buccale» en écrivant, et le lecteur aussi, même dans une réception silencieuse, parce que le langage éveillerait une articulation intérieure infime, la plupart du temps inconsciente. Ces hypothèses ont été reprises et retravaillées sous un angle psychanalytique, par exemple par Ivan Fónagy et Julia Kristeva, qui ont tenté d'établir des liens entre la phonation et un investissement pulsionnel.

Le problème de ces approches est qu'elles tendent à attribuer aux éléments sonores et rythmiques de la parole une expressivité autonome. Elles refont le dualisme du signe, dissocient le phonétique du phonologique. Même s’il admet que le rythme du discours «est d'abord un événement phonique », qu’il

35. Voir le chapitre "Critique de l'anthropologie du rythme», dans Meschonnic, 1982 , p. $642-702$.

36. Meschonnic, 1975, p. 131.

37. Meschonnic, 1982, p. 655 . 
« appartient à la sphère ORL (oto-rhino-laryngologie) 38 ", Meschonnic affirme qu'il faut éviter de concevoir le rythme « comme appartenant au monde sonore », sinon on tombe justement dans les pièges du physiologisme. L'autre effet de la réduction du rythme au sonore est celui d'entraîner la confusion entre le rythme d'un texte et une diction, «entre la réalisation phonique individuelle et l'organisation syntaxique, pausale inscrite comme organisation du texte ${ }^{39}$ ». Dans de telles conditions, par où passe alors la relation entre le rythme, le langage et le corps? Implicitement, par la voix, et l'ouïe, la mise en branle des organes de la parole, comme chez Spire, Fónagy ou Kristeva - par l'articulation, donc. Mais de même que l'articulation humboldtienne est un principe qui transcende les occurrences sonores et singulières de la parole pour devenir, dans son principe de division et de réunion, «l'essence même du langage » et, par «sa face intérieure et intellectuelle», élément de combinaisons signifiantes, de même le rythme meschonnicien procède de la composante orale du langage mais ne dépend plus directement d'une vocalisation effective. Il est «organisation, disposition » d'éléments qui à l'oral pourraient être des marques, mais qui sont inséparables de la configuration du sens: les accents et pauses sont liés à la syntaxe, au mot, ou à une organisation phonique spécifique. Irréductible au «sonore», donc, le rythme d'un texte est en fait constitué, selon Meschonnic, de «la somme des scansions possibles, pertinentes, significatives ${ }^{40} »$. Cependant, pour connaître le fonctionnement de ces marques dans une langue, il faut malgré tout passer par la réalisation vocale, par des performances: Meschonnic se sert des découvertes de la phonétique expérimentale, qui lui a fourni des informations importantes sur «la réalité orale du langage», sur ce qui fait marque dans le discours.

\section{ARTICULATION, RYTHME, SIGNIFIANT}

Le lien de l'articulation et du rythme au corps s'accompagne, autant dans la réflexion de Humboldt que dans celle de Meschonnic, de la valorisation du signifiant, qui, dans les théories issues de la «métaphysique du signe », n’a qu'un rôle accessoire. L'un des nombreux griefs de l'auteur de Critique du rythme à l'égard des théories traditionnelles est leur dimension technicienne:

Des Stoïciens à Saussure et au structuralisme [...], le langage a été avant tout vu comme un modèle réservé aux techniciens du langage, les linguistes, dans la mesure où il semblait évident que sa définition était essentiellement linguistique, c'est-à-dire

38. Dessons et Meschonnic, 1998, p. 53.

39. Ibid.

40. Meschonnic, 1982, p. 246. 
que son unité spécifique était le couplage d'un son et d'un sens - d'un signifiant et d'un signifié - tous deux constituant le signe ${ }^{41}$.

La suite de ce passage situe dans cette tradition la «double articulation » de Martinet:

Le langage est alors le seul système de signes à double articulation : des phonèmes (propres à chaque langue, comme éléments qui définissent un sens, mais qui par eux-mêmes n'ont pas de sens) et des unités de sens, les mots. Ce qu'il est, indéniablement.

Cette vision technicienne du langage [...] effaçait cependant quelque chose de capital pour la connaissance du langage: le fait que le signe, ainsi conçu, n’est pas tout le langage, mais seulement un modèle de fonctionnement $[\ldots]^{42}$.

Le fait que l'articulation est d'abord une notion de langue, et pas de discours, et le fait qu'il la voit comme un avatar du signe expliquent peut-être que cette notion n'ait pas retenu Meschonnic, même dans la pensée de Humboldt dont il se sentait si proche.

Mais le concept de Humboldt n'est pas exactement celui de Martinet. Comme le montre Trabant ${ }^{43}$, l'articulation humboldtienne est une notion plus développée que celle des linguistes d'aujourd'hui, et c'est justement elle qui, en tant qu'«essence du langage» où il n'est «rien [...] qui ne pourrait être partie et tout ${ }^{44}$ » s'oppose à une vision substitutive des unités linguistiques. Trabant expose trois grandes différences entre la notion humboldtienne et celle de la linguistique moderne. D’abord, elle n'est pas seulement division et analyse, ce qu'elle était chez Martinet; elle est, tout autant, liaison et synthèse. Ensuite, alors que Martinet s'intéressait à la différence dans le fonctionnement et la valeur de l'articulation sur les deux plans, celui des phonèmes et de la signification, Humboldt s'attache plutôt au fait que les plans sont régis par le même principe organisateur, celui de la séparation et de la réunion. Enfin, la relation entre le signifiant et le signifié est elle-même basée sur le principe de l'unité et de la division, du lien et de la coupure. Et c'est là que, justement, l'articulation devient « la raison structurale pour laquelle le mot sort totalement de la classe des signes »,

41. Dessons et Meschonnic, 1998, p. 33.

42. Ibid.

43. Trabant, 1998, p. 82-85.

44. Humboldt, 1903-1936, vol. V, p. 123. 
comme l'écrit Trabant, dans «Signe et articulation. La solution humboldtienne d'un mystère saussurien ${ }^{45}$ »:

C'est sa forme doublement articulée qui distingue le mot du signe (et de l'image de l'autre côté): l'unité indissoluble du côté matériel et du côté mental ainsi que leur distinction comme deux entités différentes sont l'effet de l'articulation qui règne comme principe structural dans les deux domaines tout en y créant des unités distinctes $^{46}$.

Étant donné ce fonctionnement, le signifiant est «partie intégrante du concept ${ }^{47}$ ». Ainsi, Humboldt affirme que «même dans le cas d'objets sensibles, les mots de différentes langues ne sont pas des synonymes parfaits et que celui qui prononce hippos, equus ou Pferd ne dit pas absolument et entièrement la même $\operatorname{chose}^{48}$ ».

Cette différence vient à la fois d'expériences (sensibles, mémorielles, imaginatives) qui s'attachent au signifiant et d'un processus associatif lié au système de la langue. Humboldt donne au moins à deux reprises l'exemple des résonances possibles du mot Wolke en allemand:

$[\mathrm{L}] \mathrm{e}$ mot prend racine dans l'imagination et le sentiment, lorsque ceux-ci sont plus vivants que l'entendement qui dissèque et dialectise. Il conserve en même temps des réminiscences symboliques secrètes, et parfois inexplicables, de l'objet qu'il désigne; ces réminiscences ne sont pas toujours directement perceptibles dans le mot luimême, mais dans ses rapports avec d'autres mots dont les objets sont semblablement éveillés par l'intuition et l'imagination, comme par exemple, en allemand, «Wolke, Welle, wehen, Wolle, weben, wickeln, wälzen, wollen» entre autres forment une série sonore manifeste ${ }^{49}$.

45. Jürgen Trabant, «Signe et articulation. La solution humboldtienne d'un mystère saussurien », Cahiers Ferdinand de Saussure, vol. 54, 2001, p. 285.

46. Ibid., p. 281.

47. Ibid., p. 277.

48. Wilhelm von Humboldt, Latium und Hellas oder Betrachtungen über das classische Alterthum, dans Humboldt, 1903-1936, vol. III, p. 170; cité par Trabant, 2001, p. 277; j'ai modifié un peu la traduction de Trabant.

49. Dans Humboldt, 1903-1936, vol. III, p. 169, et dans Humboldt, 1903-1936, vol. VI, p. 231. J'ai choisi la version de ce dernier texte: «Auf der andren Seite schlägt das Wort Wurzel in der Phantasie und dem Gefühl, wenn diese lebendiger sind, als der zergliedernde und dialectisirende Verstand. Es hat zugleich geheimnissvolle, nicht immer klar zu machende, symbolische Anklänge an den Gegenstand, den es bezeichnet, die nicht immer an diesem selbst fühlbar werden, wohl aber an solchen andren Wörtern, deren Gegenstände die Anschauung und Phantasie ähnlich anregen, so wie im Deutschen Wolke, Welle, wehen, Wolle, weben, wickeln, wälzen, wollen u. a. m. in unverkennbarem 
Dans un passage de l'Introduction à l'œuvre sur le kavi, il oppose à la vision substitutive de la signification les métaphores du maillon de la chaîne et de la touche de l'instrument:

Les hommes ne se comprennent pas parce qu'ils se remettent réellement des signes renvoyant aux choses, ni parce qu'ils se déterminent mutuellement à produire exactement et entièrement le même concept, mais plutôt parce qu'ils s'invitent mutuellement à toucher le même maillon de la chaîne de leurs représentations sensibles et de leurs productions conceptuelles internes et à frapper la même touche de leur instrument intellectuel, d'où surgissent ensuite en chacun des interlocuteurs des concepts qui se correspondent, mais qui ne sont pas exactement les mêmes. [...] Si le maillon de la chaine, la touche de l'instrument sont touchés ainsi, la totalité frémit, et ce qui surgit de l'âme en tant que concept se trouve à l'unisson avec tout ce qui entoure, jusqu'au plus lointain, le maillon individuel ${ }^{50}$.

Ces métaphores sont cohérentes avec la théorie de l'articulation: le maillon et la touche, membres à la fois séparés et liés d'une entité supérieure - la chaîne et l'instrument -, font penser aux articulations du langage: en convoquant une unité linguistique on ébranle dans l'esprit d'autres parties de celle-ci, comme on fait vibrer des harmoniques en enfonçant la touche d'un instrument ou frémir d'autres parties de la chaine en touchant un maillon. D'ailleurs, «maillon » se dit Glied, un signifiant qui fait écho à Gliederung, l'autre nom de l'articulation dans la théorie humboldtienne.

Pour Humboldt, le mot peut néanmoins être employé comme signe, de manière instrumentale: les hommes ont besoin de cet usage dans «la terminologie des sciences» et «dans les affaires de la vie pratique $e^{51}$. Mais l'usage purement substitutif du langage constitue une réduction, n’est pas le propre du

Lautzusammenhange stehn. » L'extrait de Latium und Hellas oder Betrachtungen über das classische Alterthum est plus connu en français, Caussat en ayant traduit un extrait (Humboldt, 1974, p. 19-22).

50 «Die Menschen verstehen einander nicht dadurch, dass sie sich Zeichen der Dinge wirklich hingeben, auch nicht dadurch, dass sie sich gegenseitig bestimmen, genau und vollständig denselben Begriff hervorzubringen, sondern dadurch, dass sie gegenseitig in einander dasselbe Glied der Kette ihrer sinnlichen Vorstellungen und inneren Begriffserzeugungen berühren, dieselbe Taste ihres geistigen Instruments anschlagen, worauf alsdann in jedem entsprechende, nicht aber dieselben Begriffe hervorspringen. [...] Wird nun aber auf diese Weise das Glied der Kette, die Taste des Instrumentes berührt, so erzittert das Ganze, und was, als Begriff aus der Seele hervorspringt, steht in Einklang mit allem, was das einzelne Glied bis auf die weiteste Entfernung umgiebt. » Humboldt, 1903-1936, vol. VII, p. 169-170, traduction que j'ai refaite d'après Humboldt, 1974, p. 323.

51. Trabant, 2001, p. 279. 
langage. Une réduction, parce que, grâce à l'articulation, à son "caractère ${ }^{52}$ ", grâce à son phonétisme et à son sémantisme particuliers, la langue (chaque langue) se fait «médiatrice entre les objets et les hommes» pour fixer «un monde de pensée à des $s^{5} s^{53}$ ». Cette médiation constitue même sa tâche propre. Labsence de transparence des langues d'abord, puis le fait qu'elles sont «organismes» ou «structures» $(B a u)^{54}$ où tout est lié, et enfin toujours susceptibles de transformations internes, est précisément ce qui permet la pensée, la créativité, l'existence de points de vue multiples, la subjectivité: «Du fait de la dépendance réciproque de la pensée et du mot, il est clair que les langues ne sont pas à proprement parler des moyens pour présenter une vérité déjà connue, mais, au contraire, pour découvrir une vérité auparavant inconnue $e^{55}$. Dans son essai sur l'écriture alphabétique, Humboldt explique que les écritures imagées affaiblissent «le règne de la subjectivité, l'essence du langage » - observons que Humboldt qualifie, dans le même texte, l'articulation et la subjectivité d'« essence du langage »- parce que «l'image refoule involontairement ce qu'elle veut représenter, le $\operatorname{mot}^{56}$ ». En faisant apparaître l'objet concret, elle affaiblit le caractère médiateur, sonore-idéel, du discours.

Bien qu'il ne se soit pas arrêté à la théorie de l'articulation, Meschonnic reconnaît chez Humboldt des éléments de la pensée «anti-sémiotique » qui sont inséparables de cette théorie, et qui sont tout aussi fondamentaux dans sa poétique du rythme. Je pense notamment au lien établi entre le signifiant et la dimension subjective, cognitive et créative du langage, son rôle de «médiateur entre les objets et l'homme » qui, dans l'articulation, attache « un monde de pensée à des sons $^{57}$ »: «Le rôle du signifiant dans l'interaction, l'intercréation du langage et

52. «[L]e caractère consiste dans le mode de liaison des pensées avec les sons.» Humboldt, 1903-1936, vol. VII, p. 172.

53. Ibid., vol. V, p. 101.

54. J'emploie ici «structure » pour rendre l'idée de «Bau» chez Humboldt, comme le fait Denis Thouard. Mentionnant que Humboldt lui-même utilisait le terme «structure» dans ses textes français, il explique que le linguiste désignait par ce terme ou par Bau «des ensembles de rapports relativement stables» des langues (Denis Thouard, "Glossaire » dans Wilhelm von Humboldt, Sur le caractère national des langues et autres écrits sur le langage, trad. Denis Thouard, Paris, Éditions du Seuil, coll. «Points. Essais», 2000, p. 167). Évidemment, il ne faut pas entendre la notion dans un sens structuraliste.

55. «Sur l'étude comparée des langues dans son rapport aux différentes époques du développement du langage », trad. Denis Thouard, p. 101 (Humboldt, 1903-1936, vol. IV, p. 27).

56. Humboldt, 1903-1936, vol. V, p. 111.

57. Ibid., vol. VI, p. 101. 
du référent est clairement théorisé par Humboldt ${ }^{58}$ ", écrit Meschonnic. Il fait plusieurs fois appel au passage de Latium und Hellas où apparaît la fameuse «mise en série des w», qui fournit un exemple d'une composante de sa théorie du rythme, celle de «la prosodie, [des] effets d'échos consonantiques et vocaliques ${ }^{59}$ ». Dans un texte consacré à Mallarmé, il commente la série des «W» en disant qu'il s'agissait de «[c]e que Humboldt désignait comme l'inscription du sujet dans son langage, culturellement et individuellement - l'associativité du signifiant, la subjectivité de la mémoire dans la matière des mots ${ }^{60} »$.

On se rappelle que la définition du rythme de Meschonnic est résolument énonciative, que l'organisation des marques est une «organisation du sens», de la «force» et «du sujet dans et par son discours ${ }^{61}$ ». Outre les séries associatives de phonèmes, figurent en premier lieu, parmi les marques du sujet et du rythme, les diverses formes d'accents. En français, le principal accent est syntaxique, il touche la finale des groupes. Il existe aussi un autre accent, accent initial de mot ou de groupe, qui porte plusieurs noms, et est en fait un syncrétisme de plusieurs phénomènes. Meschonnic parle d'une «accentuation prosodique», et il range dans cette catégorie tous les accents initiaux: l'accent d'attaque qui sert occasionnellement à la démarcation du groupe syntaxique; l'accent d'insistance affective ou intellectuelle; et l'accent spécifiquement "prosodique ", qui vient de la répétition de phonèmes (en particulier de consonnes) ${ }^{62}$. À cela peuvent s'ajouter les marques liées aux frontières métriques ou autres frontières formelles, le cas échéant. Les successions d'accents, ou contre-accents, ont une importance particulière, parce qu'ils vont contre le «rythme progressif» du français. Le système accentuel de la langue, et c'est particulièrement vrai pour le français, peut donner lieu, en discours, à de nombreuses réalisations: on l'a vu, Meschonnic ne veut pas noter une diction, mais la somme des possibles. Il cherche en fait une «notation progressive de la force accentuelle et des échos consonantiquesvocaliques $^{63}$ » quand plusieurs syllabes marquées se suivent, pour rendre compte des « effets croissants et décroissants d'intensité rythmique ou prosodique ${ }^{64}{ }$.

58. Meschonnic, 1975, p. 136.

59. Dessons et Meschonnic, 1998, p. 75.

6o. «Mallarmé au-delà du silence», préface à Stéphane Mallarmé, Écrits sur le livre: choix de textes, Paris, Éditions de l'Éclat, 1986, p. 40.

61. Dessons et Meschonnic, 1998, p. 75.

62. Tout ceci serait à redéfinir dans chaque langue évidemment, en fonction de son propre système phonologique et accentuel.

63. Dessons et Meschonnic, 1998, p. 44.

64. Ibid., p. 45. 
La disposition des marques dans leur ensemble crée «des figures spécifiques à un discours particulier ${ }^{65}$ ». C'est dans la création de telles figures que passe le lien entre rythme et ce que Meschonnic appelle la subjectivation du discours: celle-ci provient en effet du « fonctionnement du rythme comme la matière d'une sémantique spécifique, d'une activité qui n'est pas celle de la désignation du sens mais la constitution de séries rythmiques et prosodiques ${ }^{66} »$. Subjectivation rendue possible par le lien des marques avec la phonation et l'audition, lien enraciné dans «la matière des mots», qui porte la mémoire ou la virtualité de l'acte de parole physique. La notation progressive du rythme montre que cette subjectivation du discours va au-delà de la mesure, même dans un texte où il y a métrique, ce qu'illustre bien, entre autres exemples, l'étude que fait Meschonnic des Châtiments (1853) de Victor Hugo:

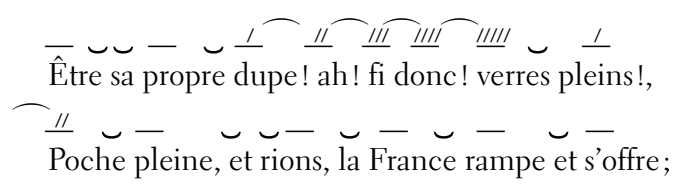

(III, XII, 20.)

La saturation accentuelle démétrifie parce qu'elle privilégie une motivation qui, en elle-même, n'est pas métrique, et qui rejoint l'accentuation dite émotive du parler. Elle favorise la mise en évidence d'une construction prosodique extrêmement serrée qui fait que l'expressivité est la résultante de tout le réseau des combinaisons prosodiques. Il n'est donc plus possible de dire que ces combinaisons sont «phoniques», sonores. Le rapprochement en série par /p/ de propre dupe, verres pleins, Poche pleine, rampe et en série par/r/ de Etre, propre, verres, rions, la France, rampe et s'offre est un rapprochement phonologique, et non plus phonétique. Il constitue un système sémantique où la valeur est partout, sans termes vides ${ }^{67}$.

Pour Meschonnic, cette notation veut aussi mettre au jour la manière dont le rythme défait le dualisme du signe et sa logique du discontinu, en créant du continu, « un système sémantique où la valeur est partout, sans termes vides ». Le «continu » et la «subjectivation » que révèle la notation des marques rythmiques sont indissociables, puisque la subjectivation se définit par «l'extension à toutes les unités d'un discours d'une qualité propre à l'invention d'un discours par un

65. Ibid., p. 150.

66. Ibid., p. 43-44.

67. Henri Meschonnic, Pour la poétique IV. Écrire Hugo, tome 1, Paris, Gallimard, coll. «Le chemin», 1977, p. 233-234. Dessons et Meschonnic ont proposé un système simplifié de «notation progressive» dans Dessons et Meschonnic, 1998. 
sujet, et d'un sujet spécifique par son discours ${ }^{68}$ ", et qu'elle "suppose la recherche d'une sémantique du continu (rythmique, prosodique) étendue à toute la systématicité d'un discours ${ }^{69}$ ». Meschonnic appelle «sujet du poème » le sujet de cette incessante production par le rythme.

En même temps qu'ils procèdent du signifiant, l'articulation de Humboldt et le rythme de Meschonnic sont donc tous deux inséparables de la subjectivité du langage. Comme le sujet de l'énonciation benvenistien (qu'ils débordent), les sujets liés à l'articulation et au rythme supposent une intersubjectivité. La subjectivation produite par le rythme n'est pas une représentation narcissique, mais une signifiance, un processus d'associations internes au discours qui engendre des valeurs spécifiques, et que le récepteur du texte reçoit, sans forcément les reconstruire entièrement et consciemment. Cette subjectivité provoque «une chaîne de réénonciations ${ }^{70} »$ :

[L]'activité du poème [qui vient du rythme] fait du texte tout entier un je, et transforme par là le je du lecteur, en sorte qu'il participe, même, encore une fois, s'il ne le sait pas, de ce je nouveau, continu, contagieux, historique et trans-historique, trans-subjectif ${ }^{11}$.

Ceci évoque la propriété qu'attribuait Humboldt au son articulé, par rapport aux cris d'animaux, à savoir l'invitation à «penser ensemble» - même si chez Meschonnic il ne s'agit pas simplement de «penser». Il faut ajouter ici que l'interlocution, la communauté de compréhension sont fondamentales dans le travail cognitif du langage selon Humboldt. Trabant voit même dans sa théorie une «troisième articulation », qu'il appelle «acroamatique ${ }^{72}$ », et qu'il reprend de manière personnelle dans ses travaux ${ }^{73}$. Il s'agit d'une articulation liée à l'oreille, qui est à la fois l'écoute de soi et celle de l'autre : elle implique elle aussi un écart

68. Ibid., p. 236.

69. Ibid.

70. Meschonnic, 1982, p. 87 .

71. Ibid., p. 192.

72. «Terme d'antiquité. Qui est reçu par l'oreille. L'enseignement acroamatique est l'enseignement oral, par opposition à l'enseignement par les livres. De là, il a pris aussi le sens de profond, le maître communiquant de vive voix à des élèves choisis un enseignement qu'il ne mettait pas dans les livres et pour l'usage du vulgaire.» Émile Littré, Dictionnaire de la langue française, disponible en ligne sur : francois.gannaz.free.fr/ Littre/xmlittre.php?requete=acroamatique\&submit=Rechercher (dernière consultation le 3 février 2011).

73. En particulier dans Trabant, 1998 et dans Jürgen Trabant, Traditionen Humboldts, Frankfurt am Main, Suhrkamp, 1990. 
(s'écouter suppose une distance de soi à soi ; écouter, une distance de soi à l'autre) et un lien. L'articulation s'étend donc même au dialogue, lui aussi constitué de séparations qui sont également des jointures:

L'écoute, qui est aussi une écoute de la pensée, est l'autre membre essentiel du mouvement articulatoire, du sien propre et de celui de l'autre. En tant qu'écoute de l'autre, l'écoute est elle-même enfin aussi, au plan macro-structurel, une jointure, articulus, et cela d'un tout que nous appelons parler-ensemble ou dialogue ${ }^{74}$.

Henri Meschonnic reconnaît dans l'idée d'une appréhension acroamatique du langage ses préoccupations pour le rythme et l'oralité:

[...] Jürgen Trabant renoue avec l'antique notion «acroamatique» du langage (de akroamai, « écouter », en grec), c'est-à-dire de l'écoute, et de l'oralité. Mais pas l'oral du signe, un son à côté d'un sens, selon l'hétérogénéité conventionnaliste du signe qui les relie ensuite dans le modèle commun. Il s'agit de rapprendre une écoute généralisée des rythmes, des rumeurs subjectives et collectives que telle œuvre littéraire, ou telle aventure culturelle, ou politique, a mises dans telle langue, tout ce qui fait qu'une langue est bien plus que de la langue au sens d'une grammaire et d'un lexique dont Humboldt disait (pourtant en plein dans le grand siècle de la grammaire historique et des dictionnaires) qu'ils ne sont que le squelette mort du langage ${ }^{75}$.

Le terme d'«écoute» revient en fait souvent sous la plume de Meschonnic, et le souci de faire sa place à une appréhension auditive de la signification - et non plus visuelle, comme la conçoivent la plupart des pensées occidentales sur le langage - est un autre trait qui le rapproche de Humboldt. Mais il ne rapproche pas l'écoute du problème de l'articulation, comme le fait Trabant.

\section{ARTICULATIONS DU RYTHME}

L'écoute nous ramène au problème de la dimension esthésique du rythme. Benveniste écrivait que le suffixe «-thmos» apportait aux notions qu'il qualifiait le sens de «modalité particulière [d]'accomplissement, telle qu'elle se présente aux

74. «Das Hören, das auch ein Hören auf das denken ist, ist das notwendige andere Glied er artikulatorischen Bewegung, der eigenen wie der des Anderen. Als Hören des Anderen ist das Hören seller schließlich auch Makro-strukturell ein Gelenk: articulus nämlich eines Ganzen, das wir Miteinander-sprechen oder dialog nennen. » Trabant, 1998 , p. 86.

75. Henri Meschonnic, «Écoutez voir», préface à Jürgen Trabant, Traditions de Humboldt, trad. Marianne Rocher-Jacquin, Paris, Éditions de la Maison des sciences de l'homme, 1999, p. XIII-XIV. 
yeux ${ }^{76} »-$ ce qui met l'accent sur le caractère sensible d'un tel accomplissement. Dans rhuthmos, c'est le fluement, l'écoulement qui est ainsi qualifié, si bien que dans son sens ancien, le «rhuthmos » était «manière particulière de fluer ».

Les séries rythmiques et prosodiques de la subjectivation, les passages de saturation accentuelle que Meschonnic met au jour à l'aide de son système de notation montrent des configurations particulières dans le mouvement, des lieux marqués qui font la spécificité de ce discours. Loin d'une métrique, ces configurations de marques créent en effet des «mouvements qui se font et se défont » dans le signifiant. À cet égard, le rythme tel que Meschonnic l'analyse se laisse bien décrire par la reformulation suivante que propose Benveniste du rhuthmos antique: “configurations" sans fixité ni nécessité naturelle et résultant d'un arrangement toujours sujet à changer ${ }^{77}$ ». Et il m’apparaît juste que le rythme discursif, hors d'un contexte métrique, se définisse ainsi. Mais je crois que cela ne suffit pas pour comprendre le rythme. Il faudrait aussi arriver à penser davantage comment on peut le dire être une «modalité particulière d'accomplissement» telle qu'elle se présente non pas seulement aux yeux, mais à notre réceptivité en général : oreille, oreille interne, en rapport ou non avec une prononciation, avec ou sans voix, avec ou sans conscience claire, dans la lecture ou l'audition. Et cela même dans la perspective meschonnicienne de la notation d'une organisation, de la somme des scansions possibles - qui ne peuvent être toutes réellement perçues. Il s'agit d'imaginer comment le rythme, «manière particulière de fluer » fait appelle à l'esthésis, peut être reçu - par les sens, par l'intelligence, inséparablement - sans postuler que tout soit effectivement perçu consciemment. Dire que le «flux» s'effectue selon un mode particulier suppose sa qualification par des éléments qui s'y différencient, y font saillie: les «marques », accents et séries, en font office. Mais cela suppose aussi du particulier dans le mouvement même, ou plutôt, la possibilité d'appréhender un processus spécifique de formation - ce que Meschonnic n’aborde pas pour les raisons déjà exposées.

Dans l'élaboration de sa théorie, Humboldt s'attachait plus concrètement que l'auteur de Critique du rythme à la dimension sensible et perceptive de l'articulation. Il a cherché à expliquer son hypothèse selon laquelle le son et l'ouïe sont respectivement la matière et le sens les plus adaptés à l'articulation, et donc à la formation du langage, comme il le formule si bien dans le passage que j’ai mis en exergue de ces pages: «La liaison indissoluble de la pensée, des organes de la voix et de l'ouïe avec le langage se trouve irrévocablement

76. Ibid., p. 332 .

77. Benveniste, 1966, p. 333 . 
dans l'organisation originaire de la nature humaine, qui ne peut être expliquée davantage $^{78}$.» Pour étayer cette affirmation, Humboldt essaie de montrer, par une série de comparaisons, de quelle manière le déploiement matériel, sonore et extérieur de la parole fournit un «portrait» sensible du cours immatériel, intérieur et en soi imperceptible de la pensée ${ }^{79}$. Dans ces comparaisons on retrouve le principe de division et de réunion (ou synthèse) de l'articulation : le son convient bien à la réflexion, parce qu'il est seul capable de retentir en « une netteté et une unité bien détachées ", tout comme la pensée, «comparable à un éclair ou à un coup, rassemble toute la faculté de représentation en un point, et fait abstraction de tout ce qui survient en même temps »; l'entendement peut «synthétiser une série de pensées en des unités quelconques », ce que font aussi «l'imagination et l'oüie avec une série de sons ${ }^{80} »$. C'est donc parce qu'elle peut aussi effectuer une opération de division et de synthèse ${ }^{81}$ que «l'oreille est le seul sens qui soit bien adapté à l'articulation ${ }^{82}$ ». Mais c'est aussi, en même temps, parce qu'elle peut percevoir «l'impression produite par le mouvement, et dans le cas du son que fait résonner la voix, percevoir l'impression produite par une véritable action ${ }^{83}$ ». Elle le peut comme aucun autre sens, parce qu'elle saisit quelque chose de linéarisé, alors que dans la vue plusieurs choses en même temps peuvent se brouiller. Le caractère mobile, temporel, du son proféré est aussi ce qui le rend propre à s'unir avec la pensée.

En tant qu'ensemble de tensions entre liaisons et séparations qui composent tous les niveaux du discours, en tant que processus où le mouvement est constamment organisé par «le fait que [la forme de la pensée et l'articulation du son] divisent leur domaine en éléments constitutifs dont l'assemblage ne donne que des totalités ayant elles-mêmes tendance à devenir des parties de nouvelles totalités ${ }^{84}$ ", l'articulation contient en germe une possible «organisation

78. Voir note 1 pour la référence.

79. Voir notamment Humboldt, 1903-1936, vol. VI, p. 154 et ibid., vol. VII, p. 54. La comparaison est plus développée dans le texte plus ancien.

8o. Ibid., vol. VI, p. 154 .

81. Voir à ce sujet Trabant, 1998 , p. 87 .

82. «In der Wirklichkeit ist das Ohr der ausschliesslich für die Articulation bestimmte Sinn », Humboldt, 1903-1936, vol. VI, p. 154.

83. «Es beruht dies sichtbar darauf, dass das Ohr (was bei den übrigen Sinnen nicht immer oder anders der Fall ist) den Eindruck einer Bewegung, ja bei dem der Stimme entschallenden Ton einer wirklichen Handlung empfängt [...].» Ibid. (je souligne).

84. «Dasjenige, worin sich diese Form und die Articulation, wie in einem verknüpfenden Mittel begegnen, ist, dass beide ihr Gebiet in Grundtheile zerlegen, deren 
du mouvant». Si on se représente les «totalités» emboîtées comme des groupements, des ensembles qui se forment par la synthèse, mais aussi par la division, en se séparant d'autres groupes, on a presque une définition minimale du rythme. On pense au psychologue Paul Fraisse, qui voyait dans le rythme la différenciation d'un continuum fonctionnant par assimilation et distinction des durées: «Les différences entre des éléments voisins par la durée, la longueur, la forme sont diminuées ou supprimées par assimilation et les différences notables sont exagérées, ce qui supprime toute équivocité de la structure différenciée ${ }^{85}$.»

La définition de Fraisse est toutefois abstraite et formelle, elle est séparée du langage, et donne raison à Meschonnic suivant lequel tenir compte de la dimension perceptive du rythme conduirait à séparer la forme et le sens. Mais l'articulation humboldtienne n'est pas la définition de Fraisse. Elle explique le fonctionnement fondamental du langage «qui attache un monde de pensées à des sons ». Ce fonctionnement fait le pont entre la notion de langue et celle de discours. Tout processus de synthèse et d'analyse y est lié à de la signification, ne serait-ce qu'à l'état naissant. L'articulation n'est toutefois pas suffisante non plus pour définir le rythme: la notion demeure trop abstraite: tout, dans le langage, est divisé et lié à tous les niveaux; on ne touche pas encore le particulier d'une manière de fluer. Il faut retenir de l'articulation, entre autres, le principe d'une tension entre continu et discontinu, réunion et séparation, rapprochement et écart, pour les intégrer à une notion du rythme comme organisation du signifiant. Dans sa référence au rhuthmos héraclitéen, Meschonnic met toujours l'accent sur le continu, contre le signe et la métrique ${ }^{86}$. Plus encore, il décrit le continu comme un mouvement qui fait du discours un «plein», un flot qui «emporte et déborde ${ }^{87} »$ :

Il n'y a plus alors un signifiant opposé à un signifié, mais un seul signifiant multiple, structurel, qui fait sens de partout, une signifiance (signification produite par le signifiant) constamment en train de se faire et de se défaire, de l'unité à l'élément

Zusammenfügung lauter solche Ganze bildet, welche das Streben in sich tragen, Theile neuer Ganze zu werden. » Ibid., vol. VI, p. 152.

85. Paul Fraisse, Psychologie du rythme, Paris, Les Presses universitaires de France, coll. «Le psychologue», 1974, p. 111-112.

86. Même sa lecture de Humboldt va dans ce sens, comme le dit Trabant: "Meschonnic invoque Humboldt dans le contexte du concept de "continu” qu'il oppose au discontinu», et il ajoute: «[m]ais je ne trouve pas vraiment de passage humboldtien opposant le continu au discontinu». Trabant, 2005, p. 180.

87. Dessons et Meschonnic, 1998, p. 56. 
qui peut à son tour être une unité-valeur momentanée dans le système d'un discours, et en interaction avec la signification contextuelle ${ }^{88}$.

Mais pour qu'apparaisse, dans le flux héraclitéen, une «modalité particulière d'accomplissement, telle qu'elle se présente aux yeux» ou à d'autres modes de saisie comme l'ouïe, il faut que s'y disposent des éléments différenciateurs, ce que sont les marques et les pauses. Les marques n'organisent pas seulement une signifiance, mais, tout comme les pauses, elles produisent un découpage dans le flux, des groupements et des intervalles, une forme de discontinuité ${ }^{89}$. Pour que l'on puisse appréhender la spécificité de tel ou tel déroulement, il faut que s’instaure un processus de comparaison - des synthèses - entre des phases du flux, entre des points qualifiés et les divers niveaux de groupements qu'ils créent.

Or, les marques rythmiques et les pauses surviennent toujours à un certain niveau de l'articulation au sens humboldtien, et même plus, elles sont ellesmêmes des articulations - des «jointures» : les accents prosodiques sont issus soit d'une organisation particulière des phonèmes, soit de la mise en relief d'un mot (pour les accents dits affectifs ou intellectuels) ou d'un syntagme (accent d'attaque); les accents toniques sont liés à la syntaxe; les pauses, à des unités syntaxiques ou énonciatives. Et tous ces lieux marqués - qui sont des passages du corps et des liaisons de signifiants - forment la subjectivation du discours. Si on reprenait cela dans les termes de l'articulation humboldtienne, on pourrait dire que, quand la séparation et la liaison organisent un découpage spécifique, quand certains lieux de séparation ou de synthèse hiérarchisent le principe général de réunion et de séparation en devenant des marques et suscitent une écoute associative -, alors on accède au rythme.

88. Meschonnic, 1975, p. 512.

89. Même si, dans la parole concrète, il n'y a pas toujours d'arrêt de la voix là où on « entend» des pauses. 